International Journal of Geography and Geology

2014 Vol. 3, No. 5, pp. 68-77

$\operatorname{ISSN}(e): 2305-7041$

$\operatorname{ISSN}(p): 2306-9872$

DOI: $10.18488 /$ journal.10/2014.3.5/10.5.68.77

(C) 2014. Conscientia Beam. All Rights Reserved.

Cross Mark

\title{
INVERSION OF ELECTRICAL RESISTIVITY DATA BY VES METHOD FOR WATER EXPLORING CASE STUDY: SOUTH WEST OF HAMEDAN, IRAN
}

\author{
Ahmad Alvandi ${ }^{1}$--- Mojtaba Babaei ${ }^{2}$ \\ ' Young Researchers and Elite Club, Toyserkan Branch, Islamic Azad University, Toyserkan, Iran \\ ²Department of Geophysics, Hamedan Branch, Islamic Azad University, Hamedan, Iran
}

\begin{abstract}
The purpose of this research is to explore the water in the south west of Hamedan which has many environmental problems and very few water resources. Also in the area, due to the lack of awareness, the farmers excavate the wells with high cost that results in declining water table. The collected data was investigated via the electrical probing at the Schlumberger array and Jointed with the wells data and geological information. The data processing was performed by conventional methods of calculation and utilized specialized software WINSEV, RES2D, RES3D, 2WIN at direct and inversion states. Finally the results of the research are acceptable and pleasant.
\end{abstract}

Keywords: Specific electrical resistance method, Schlumberger array, Yekn-Abad of Hamedan, Inversion.

Received: 13 April 2014/ Revised: 7 May 2014/ Accepted: 12 May 2014/ Published: 16 May 2014

\section{Contribution/Originality}

Groundwater potential aquifers producing zones have been delineated through investigation conducted by the electrical resistivity survey. Good prospects therefore exist for groundwater development in the study area where the depth to basement is relatively thick and has favorable low resistivity, while those with thin depth to bedrock and high resistivity value have a lower potential for an aquifer.

\section{INTRODUCTION}

Geoelectrics studies deal with the detecting of surface effects that are the results of passing the current across earth. This method compares with other geophysical methods; such as gravity, magnetic, elastic and radioactivity in which only a force field or anomaly property was used. By electrical methods, comma in between potential, currents and electromagnetic fields that are 
naturally or artificially in earth, were determined. In addition, the measurements can be performed by different methods and their results were different, too. In fact, due to changes in the electrical conductivity of rocks and minerals, it is possible to use these methods.

Accordingly (except specific resistance) are the component of natural springs and the others are sorted as artificial springs. In the study, the specific resistivity method was used to identify the status of hydrology and groundwater resources of the area; which should recognize geological strata, natural and artificial structures which are likely buried; the effects of tectonic and determining depth at the floor stone. It was selected on the basis of conditions of the region. Due to variety and useful application of this method, it is mostly used in geological studies outside the context of petroleum studies.

\section{ELECTRICAL RESISTIVITY METHOD}

In the electrical resistivity method, direct current (DC) or alternating current (AC) was entered in the earth with too low frequency by current electrodes and potential differences were measured by a pair potential electrode. The electrical current of single electrode was illustrated in figure 1 at the homogenous and isotropic area with certain specific resistivity. The electrical current scattered in the earth like hemisphere and the isopotential surface was calculated in the "p" point which was located at distance of " $\mathrm{r}$ " from the current electrode. "I" current was distributed at the hemisphere surface area of which was $2 \pi \mathrm{r}^{2}$. So the current density is equal to:

$$
\mathrm{J}=\frac{\mathrm{I}}{\mathrm{A}}=\frac{\mathrm{I}}{2 \pi \mathrm{r}^{2}}
$$

Fig-1. The electrical current lines and isopotential surface around the single electrode

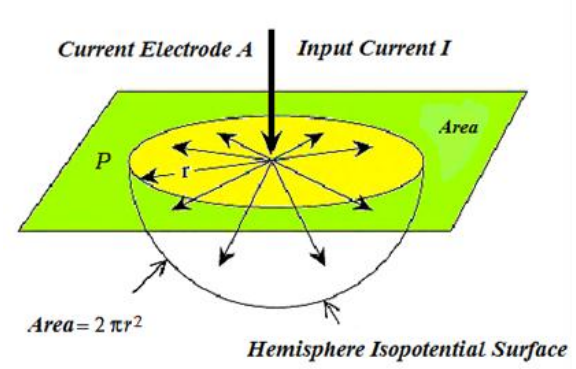

At first, the minor changes of potential were related to current density, calculated at "p" point. If the potential drop is assumed " $\mathrm{V}_{\mathrm{r}}$ " at the "p" point (with respect to point $\mathrm{A}$ ), also the potential drop in a very small distance from this point is as " $\mathrm{dV}$ ", potential difference between two points is equal to:

$$
\mathrm{dV}=-\frac{\rho \mathrm{I}}{2 \pi} \frac{\mathrm{dr}}{\mathrm{r}^{2}}
$$

To calculate the total potential drop at the point "p" with respect to point " $\mathrm{A}$ ", it is sufficient that the equation $(2)$ is integrated over the distance $r$. So: 


$$
V_{r}=\int d V=-\int \frac{\rho I}{2 \pi} \frac{d r}{r^{2}} \rightarrow V_{r}=\frac{-\rho I}{2 \pi} \frac{1}{r}
$$

With the equation (3), the potential at every point (at or below the surface) of a homogeneous and isotropic medium can be calculated. If two current electrodes "A" \& "B" and two potential electrodes "M" \& "N" were assumed in the ground surface (Fig. 2), the potential differences between two electrodes can be calculated by equation (4).

$$
\Delta \mathrm{V}_{\mathrm{MN}}^{\mathrm{A} \cdot \mathrm{B}}=\mathrm{V}_{\mathrm{M}}^{\mathrm{A} \cdot \mathrm{B}}-\mathrm{V}_{\mathrm{N}}^{\mathrm{A} \cdot \mathrm{B}}=\frac{\rho \mathrm{I}}{2 \pi}\left\{\frac{1}{\overline{\mathrm{AM}}}-\frac{1}{\overline{\mathrm{BM}}}-\frac{1}{\overline{\mathrm{AN}}}+\frac{1}{\overline{\mathrm{BN}}}\right\}
$$

Fig-2. General form of the potential and current electrodes arrangement

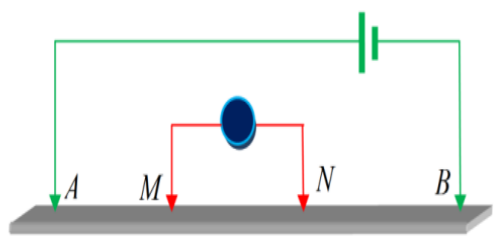

The equation (4) can be rewritten as follows:

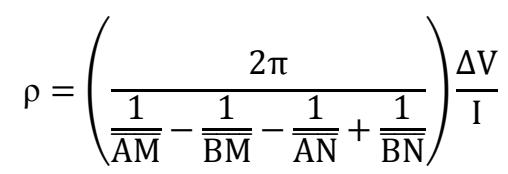

The term in brackets of equation (5) is called the geometric factor arrangement of electrode and usually indicated by K. so It becomes:

$$
\rho=K \frac{\Delta V}{I}
$$

If $\rho$ is measurement in a homogeneous and isotropic medium, it is called the real specific resistivity. But in the heterogeneous and anisotropic area, it is named apparent specific resistivity. The apparent specific resistivity is a function of the following variables.

I. Distance between the electrodes

II. Geometrical arrangement of electrodes

III. Real specific resistivity and other subsurface material properties such as thickness and slope layers (Emenike, 2001).

\subsection{Electrode Arrays}

The common arrays of the resistivity method are based on using four electrodes. The "A" \& "B" electrodes are for sending current to the ground and "M" \& "N" electrodes are applied to determine the potential drop in the ground. According to position states of the electrodes relative 
to each other, the current magnitude and voltage value, the apparent specific resistivity can be estimated.

Fig-3. The common electrode arrays in the geo-electrical exploration (Denchik et al., 2005)

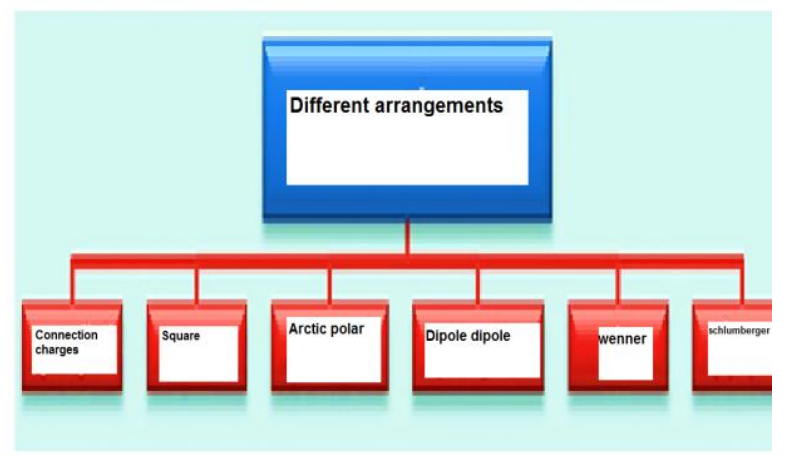

Schlumberger array is widely used in the electrical explorations. Four electrodes (B, N, M \& $\mathrm{A}$ ) is at straight line on the ground as figure 2 . In this array, the distance between A \& B is equal or greater than 5 time distance between $\mathrm{M} \& \mathrm{~N}$. in this arrangement, the value of $\rho_{S}$ is equal to:

$$
\rho_{s}=\pi \frac{(\overline{A B} / 2)^{2}-(\overline{M N} / 2)^{2}}{\overline{M N}} \frac{\Delta V}{I}
$$

\section{GEOLOGY OF AREA}

The study area is located the vicinity of Sanandaj-Sirjan zone. The zone has a common history with central Iran, but the structure, direction and gradient of layers are similar to the Zagros. Major rock units in this region are as follow:

Shale and sandy limestone (TRs): includes shale thickness of which is $100 \mathrm{~m}$, interbed layer of it is sandylimestone with crystalline. The age of this unit is Triassic and it has low permeability.

Quartzite, shale and sandstone $(\mathrm{Jq})$ : Includes Quartzite and shale is with thickness of 500 meters. Within this unit, layers of limestone, sandstone and rarely volcanic rocks are seen. The transmissivity and permeability of unit is very important. Its age is the Lower Jurassic.

Basalt (KJ): The scope of the project, units of basaltic volcanic rocks can be seen. The volcanic rock indicates onset of volcanic activity at the end of the Jurassic period.

Silty shales and sandstones (K1): the mountain of South and South-West Area consists of shale units which are slightly metamorphosed. Chlorite and epidote minerals are seen within the shale. The type of sandstone is quartzite and it is extremely hard. It contains more mica and chlorite

. The age of unit is Lower Cretaceous, it is less important to hydrogeology.

Red sandstone and conglomerate (K2): This unit has a thickness of about 131 meters. It has been very diverse type of sand such as: shale, sandy shale, red conglomerate, quartzite and sandy limestone. age of the unit is Cretaceous. Water transmissivity (permeability) properties of unit are considered unimportant. 
Orbitulina limestone (K3): The thickness of unit is 300 meters, this unit is marly limestone, yellow to gray dolomite and marly limestone at base of formation, contains organic material and chert grains in limestone at the top of the unit. The boundary with older rocks have same slope. The age of its fossils is Lower Cretaceous. According to the kind of materials, the transmissivity coefficient is low.

Silty clays, chalk, marly sandstone and sandstone $(\mathrm{Pm})$ : the type of material is variety including clays, silt, chalk, marly sandstone, sandstone, conglomerate, chalky limestone. Some horizons of it can have erosion and Help in reducing to reduce the height. The age of unit is Pliocene. It seems that this unit does not have the ability to form an aquifer.

Upland (QT), Travertine (Qt), fluvial deposits (Qr), alluvium (Qal): In many parts of the research area area, discontinuous deposits are formed from different materials. Fluvial deposits are the recharge zone of the aquifer and base flow of rivers or flood flows in these ducts. The main aquifer in the alluvium plain is formed.

\section{FIELD OPERATIONS}

Considering the objectives of the project and local geological conditions, electrical probing method was used with the Schlumberger array which is an applied array and geoelectrical method

. In the field, some instruments were used such as: Akson Resiver model SA300C machine with one neutralization SP automatic connection, switching system, anti-noise cable, and converter with the ability to produce variable voltage and constant up to the maximum 500 volts and 12 volts battery, standard bronze electrode, pulleys, etc. Overall, the amount electrical probing were 41 which were into 4 longitudinal profiles as appropriate. So the whole area was covered (Fig. 4).

Fig-4. Local probing position for exploration of water

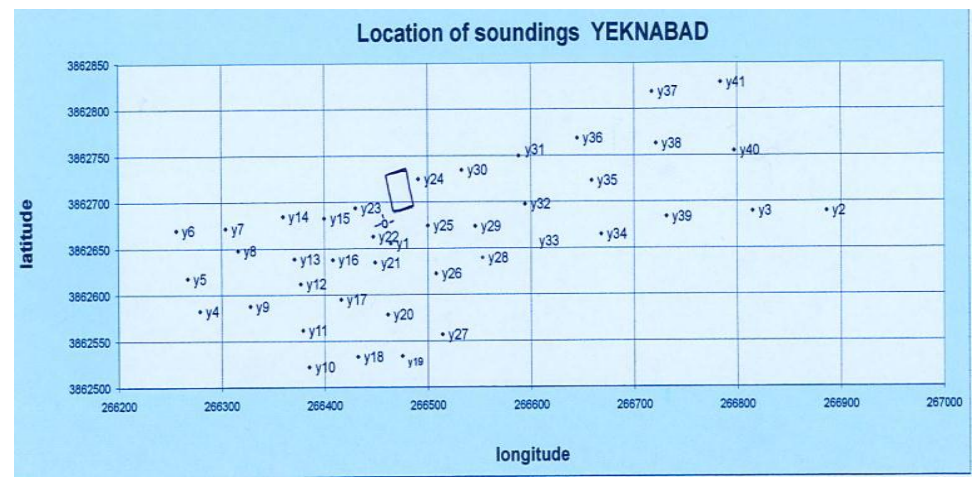

\section{THE STUDY AREA}

The study area is located in the border of Hamedan city. Its longitude is between 266,200 and 267,000 and latitude coordinates is between 3862500 and 3862850 . Also, the area is equivalent to about 120,000 square meters. 


\section{DATA PROCESSING}

After collecting and Collection of data, Data was processing with charts $2 \mathrm{~L}, 3 \mathrm{~L}$ and conventional methods of computing. At the same time, collected data was processed with using the WINSEV, RES2D, RES3D and 2WIN software, inversion and directly methods. After the results of both methods were compared and have been Re-corrected and processed. again (Savvaidis et al., 1999)]. Finally, thereby, The most accurate results were obtained (Fig. 5).

\subsection{Apparent Specific Isoresistivity Plans}

In order to demonstrate the total conductivity of earth at various depths, the apparent resistivity was calculated at various depths. For example, a model of this type of plan that you have displayed the depth of 7 meters (Figure 6). This is a suitable way to compare apparent resistivity of different sections at certain depth than apparent resistivity at around of the certain depth.

These changes in resistance will be studied at the next stages and causative agent would be determined. In this step, twelve plans had been investigated at various depths. It should be noted that the depth listed in the plan, each compared to the same point of the surface and according to the topography of the area, the point at the surface may not be flat surface. In the plans, the blue area has lower apparent resistivity than the red area (Fig. 6) (Ward, 1990).

Fig-5. Data processing pattern in the electric resistivity method (Reinhard, 1974)

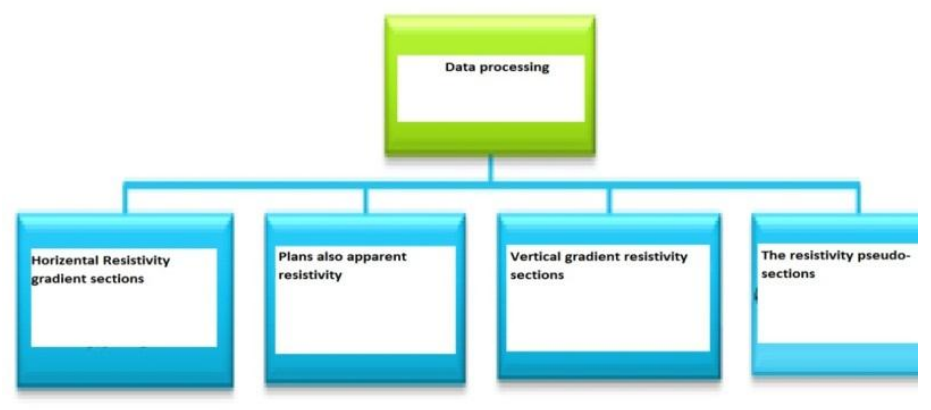

Fig-6. Apparent isoresistivity Plans at a depth of 7 meters (Yekn-Abad of Hamedan)

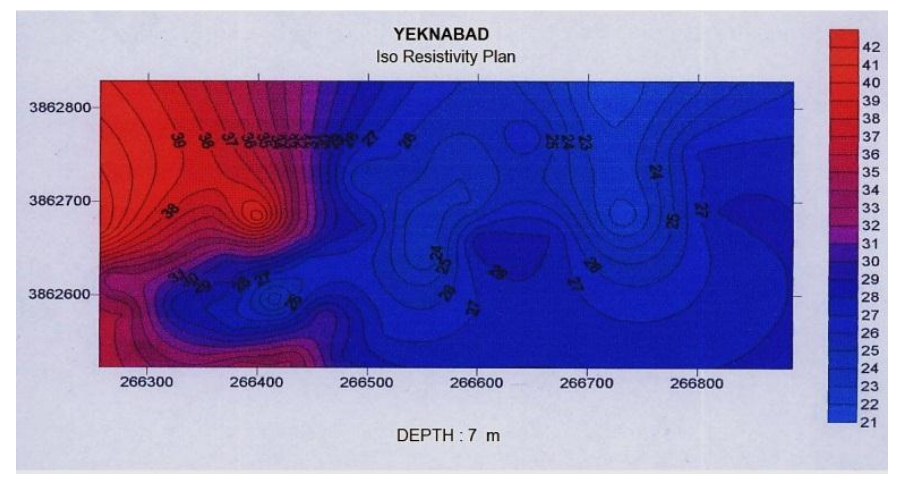




\subsection{The Pseudo-Cross Sections of Isoresistivity}

In these sections, the overview of changes in apparent resistivity are shown until the define depth. The general useful information as schematic of the resistivity at different depths can be obtained by accurate investigation of apparent resistivity changes in the sections. In this part, the apparent resistivity pseudo-section is shown as an example (Fig. 7).

Fig-7. The typical pseudo cross section of apparent isoresistivity, y3-y9.

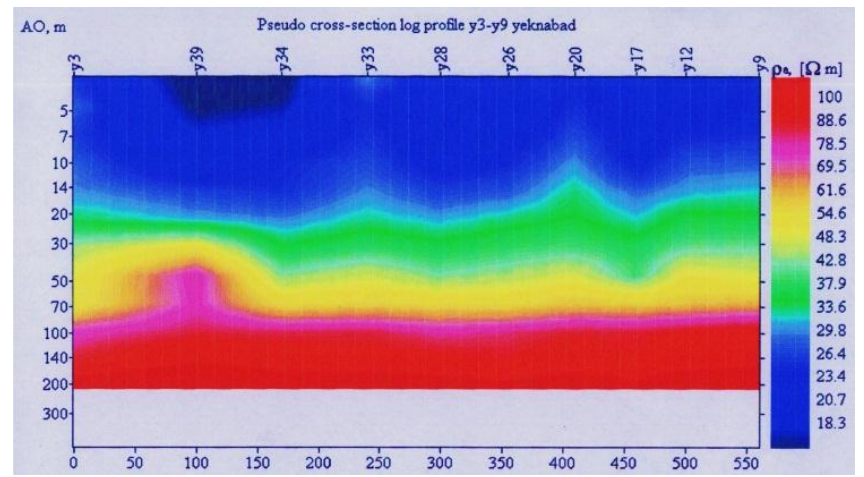

\subsection{The Pseudo-Cross Sections of Vertical Gradient Resistivity}

In the sections, the values of vertical gradient apparent resistivity are illustrated schematically. The pseudo-sections give some very useful information about horizontal layers and gradient layers and some geological structures. Yeknabad, axis of depth is shown as logarithmic (Fig.8).

Fig-8. The typical pseudo crass section of vertical gradient apparent resistivity, y6-y41

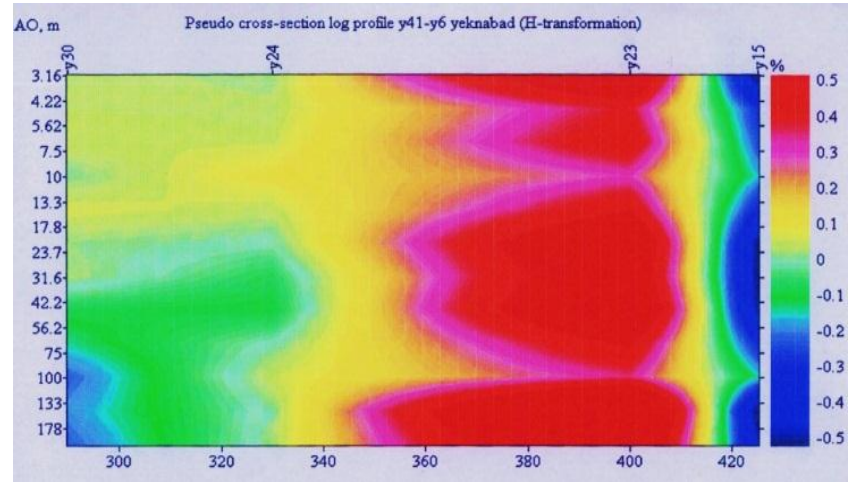

\subsection{The Pseudo-Cross Sections of Horizontal Gradient Resistivity}

In the cross-section, horizontal changes of resistivity are displayed. To locate contact, faults and any structural that cause adverse changes to the resistivity gradient, it is easily possible by using these sections (Fig. 9). 
Fig-9. The typical pseudo cross section of horizontal gradient apparent resistivity, y 8-y 40

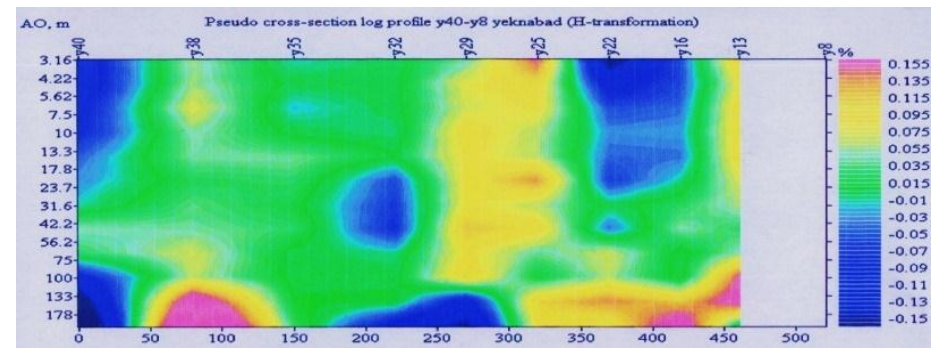

\subsection{Data Interpretation (Section of Electrical Isoresistivity)}

After the field data processing and converting the apparent resistivity to the real resistivity, data was interpreted by combining the data that was obtained via previous results and regional geological data. The results of it are of accurate geological sections from Yekn-Abad that have Comprehensive information of geological strata. Also at this stage, the reasons of items listed in the data processing are specified. In the part, the four resistivity profiles of the region are provided while location of profiles and the layers have been diagnosed, are described.

Resistivity profile "1": This profile contains 12 Schlumberger electrical probing; its current transmitter with a maximum is 1000 meters. Profiles' length is $600 \mathrm{~m}$ and the direction is eastwest (Fig.10).

Fig-10. Resistivity profiles (1), the vertical axis shows the depth and the horizontal axis is extent (yeknabad)

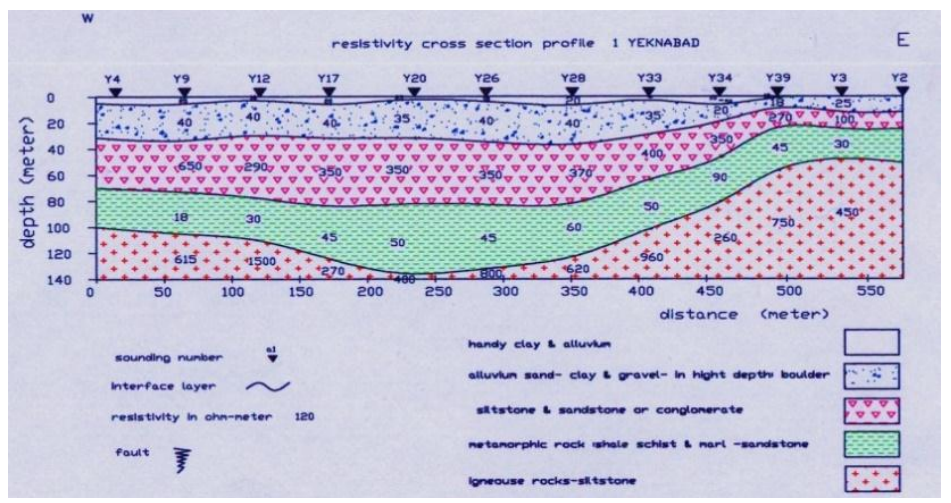

Resistivity profile "2": This profile contains 13 Schlumberger electrical probing; its current transmitter with a maximum is 1000 meters. Profiles' length is $600 \mathrm{~m}$ and the direction is eastwest.

Resistivity profile "3": This profile contains 11 Schlumberger electrical probing; its current transmitter with a maximum is 1000 meters. Profiles' length is $600 \mathrm{~m}$ and the direction is eastwest.

Resistivity profile "4": This profile contains 4 Schlumberger electrical probing; its current transmitter with a maximum is 1000 meters. Profiles' length is $100 \mathrm{~m}$ and the direction is eastwest (Fig. 11). 
Fig-1 1. Resistivity profiles (4), the vertical axis shows the depth and the horizontal axis is extent (Yeknabad)

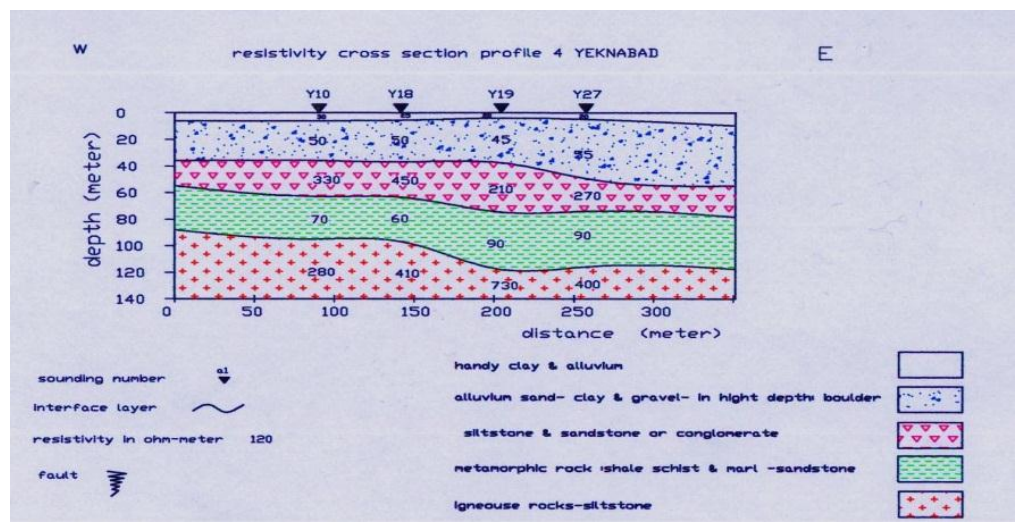

\subsection{Interpretation of Data on the Profiles Has the Same Results As Follows}

The first layer includes soil and alluvial clay and fine-grained which has Varying electrical resistivity. Its average thickness is $5 \mathrm{~m}$.

The second layer includes alluvial sediment and fine-grained with a little gravel which has Varying electrical resistivity. The Upper layer is vadose and the lower is saturated but the thickness of saturation zone is very small. Its average thickness is $40 \mathrm{~m}$.

The third layer is relatively thick and resistant. This layer is made of eroded and metamorphic rock, siltstone, sandstone and conglomerate. The strength of the rock layers in the upper is depth than in the deep, so the up of layer has many fractures and some water in there but in the depth, the fractures concentration is low.

The fourth layer is of relatively low-resistivity. The thickness is about $10 \mathrm{~m}$. This layer is made of metamorphic rock formations and possibly shale, marl and loose conglomerate schist. Unlikely that there is a wet layer.

The fifth layer is Resistant and impermeable and consist of igneous rock. The formation is close to the surface in the eastern part.

\section{CONCLUSION}

The study area includes a portion of the fine to medium grained alluvium in the surface; middle part is weathered rock and in depth it has the interbed layers of schist and marl. The saturated zone is located in the lower alluvial layer at the depth of approximately $30 \mathrm{~m}$. It has the low thickness and penetrated in a small portion of the rock. Large tectonic effects have not been observed in the region, fractures and fault haven't been detected. The only problem is the uplift resistance layer in the eastern part of the area. Changes of bed rock depth are very limited in most parts of the area. According to the resistivity trend, the general direction of groundwater flow is to the north and northeast of the study area. Under all conditions, low depth of bedrock and low thickness of aquifer, the amount of aquifer storage is very small and the probability of high water extraction is very low. 
Funding: This study received no specific financial support.

Competing Interests: The authors declare that they have no competing interests.

Contributors/Acknowledgement: All authors contributed equally to the conception and design of the study.

\section{REFERENCES}

Denchik, N., L. Marescot and D. Chapellier, 2005. Choice of effective electrode array for 3D electrical resistivity monitoring during rainfalls. Institute of Geoph Ysics, University of Lausanne, College Propedeutique.

Emenike, E., 2001. Geophysical exploration for ground water in a sedimentary environment. Global J. Pure and Applied Sci., 12: 323-326. [Accessed Jan 1-3].

Reinhard, K., 1974. Combined geo-electrical and drill-hole investigation for detecting fresh-water aquifers in Northern Western Missouri. Geophysics, 39: 340-352.

Savvaidis, A., G. Tsokas, P. Soupios, G. Vargemezis, M. Manakou, P. Tsourlos and I. Fikos, 1999. Geophysical prospecting in the krousovitis dam (N Greece) by seismic and resistivity geophysical methods. J. Balkan Geophys. Soc., 21: 28-39.

Ward, S.H., 1990. Resistivity and induced polarization methods geotechnical and environmental geophysics ed sward. Tulsa, OK: Society of Exploration Geophysicists. pp: 147-89.

\section{BIBLIOGRAPHY}

Dahlin, T. and C. Bernstone, 1997. A roll-along technique for 3D resistivity data acquisition with multi electrode arrays. Proceedings- SAGEEP'97 (Symposium on the Application of Geophysics to Engineering and Environmental Problems), Reno, Nevada, March 23-26., 2: 927-935. 